\title{
Feasibility of automated detection of sleep apnea using implantable pacemakers and defibrillators: a comparison with simultaneous polysomnography recording
}

\author{
Domenico G. Della Rocca ${ }^{1}$ (D) Maria Albanese ${ }^{2} \cdot$ Fabio Placidi $^{2} \cdot$ Giovanni B. Forle $^{3} \cdot$ Luigi Di Biase $^{1,4,5,6}$.

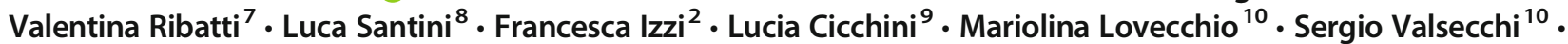 \\ Carlo Lavalle $^{11}$ - Andrea Natale ${ }^{1,5,12,13,14,15}$ - Nicola B. Mercuri ${ }^{2,16}$. Andrea Romigi ${ }^{17}$
}

Received: 22 August 2019 / Accepted: 16 September 2019/Published online: 23 October 2019

(C) Springer Science+Business Media, LLC, part of Springer Nature 2019

\begin{abstract}
Purpose Sleep-related breathing disorders (SRBD) have been associated with adverse cardiovascular events and prognosis. Some modern pacemakers (PMK) and implantable cardioverter defibrillators (ICD) are equipped with thoracic impedance monitoring systems which allow detecting fluctuations in tidal volume occurring in SRBD. This study aimed at assessing the accuracy of a novel transthoracic impedance-based PMK/ICD sensor for automatic detection of SRBD.

Methods We prospectively enrolled 31 consecutive patients scheduled for implantation of a PMK or an ICD with sleep respiratory disturbances monitoring capability. The results of unattended home nocturnal recordings expressed as apnea/hypopnea index (AHI) were compared with the respiratory disturbance index (RDI) stored in the cardiac device memory at the time polygraphy was performed.

Results Among the 31 enrolled patients ( 20 men, mean age $70 \pm 8$ years), a comparison between the data from polygraphy and cardiac device was feasible in 29 patients. According to the results of home nocturnal recording, a severe SRBD was observed in $7 / 29$ (24.1\%) patients. On the basis of receiver-operating characteristic curve analysis of RDI values, the optimal RDI cutoff value to identify severe SRBD was 47 episodes/h (sensitivity $100 \%$, specificity $100 \%$ ). Bland-Altman agreement analysis of AHI-RDI revealed a bias between measurements of -11 episodes $/ \mathrm{h}$, with limits of agreement -38 to 15 episodes $/ \mathrm{h}$.

Conclusions In the present study, the novel transthoracic impedance-based monitoring system ApneaScan ${ }^{\mathrm{TM}}$ appeared effective in screening PMK and ICD patients for SRBD.
\end{abstract}

Domenico G. Della Rocca and Maria Albanese contributed equally to this work.

Domenico G. Della Rocca

domenicodellarocca@hotmail.it

1 Texas Cardiac Arrhythmia Institute, St. David's Medical Center, 3000 N IH-35, Suite 720, Austin, TX 78705, USA

2 Sleep Disorders Centre, Neurophysiopathology Unit, Department of Systems Medicine, University of Rome "Tor Vergata", Rome, Italy

3 Azienda Ospedaliera - Polo Universitario - "Luigi Sacco", Milan, Italy

4 Albert Einstein College of Medicine, Montefiore Hospital, New York, USA

5 Department of Biomedical Engineering, University of Texas, Austin, TX, USA

6 Department of Clinical and Experimental Medicine, University of Foggia, Foggia, Italy

7 Cardiac Arrhythmia Research Center, Centro Cardiologico Monzino, IRCCS, Università di Milano, Milan, Italy
G.B. Grassi Hospital, Ostia, Rome, Italy

9 Department of Cardiovascular Medicine, University of Tor Vergata, Rome, Italy

10 CRM Department, Boston Scientific Italy, Viale Forlanini 23, 20134 Milan, Italy

11 Department of Cardiovascular, Respiratory, Nephrology, Anesthesiology and Geriatric Sciences, "Sapienza" University of Rome, Policlinico "Umberto” I, Rome, Italy

12 Interventional Electrophysiology, Scripps Clinic, La Jolla, CA, USA

13 Metro Health Medical Center, School of Medicine, Case Western Reserve University, Cleveland, OH, USA

14 Division of Cardiology, Stanford University, Stanford, CA, USA

15 Electrophysiology and Arrhythmia Services, California Pacific Medical Center, San Francisco, CA, USA

16 IRCCS Santa Lucia Foundation, Rome, Italy

17 IRCCS Neuromed, Via Atinense, 18 Pozzilli, IS, Italy 
Keywords Pacemaker $\cdot$ Sleep apnea $\cdot$ Sleep apnea screening $\cdot$ Transthoracic impedance $\cdot$ Implantable defibrillator

\section{Introduction}

Over the past decade, raising awareness of the role of sleep-related breathing disorders (SRBD) in the causation and promotion of several cardiovascular diseases has led to a significant increase in evaluation of sleep respiratory disturbances [1]. Specifically, SRBD have been demonstrated to significantly increase the risk of developing hypertension [2] and arrhythmias [3, 4], as well as worsen outcomes in patients with heart failure [5], being a predictor of premature cardiovascular death. Nonetheless, SRBD screening is not yet integrated into current clinical practice and remains largely underdiagnosed by means of standard monitoring technology.

Thoracic impedance monitoring systems have been developed in order to detect fluctuations in tidal volume occurring in SRBD [6-8]. Some modern pacemakers (PMK) and implantable cardioverter defibrillators (ICD) are equipped with these algorithms, which allow for continuous monitoring of the severity of sleep apnea (SA). Although SRBD detected and monitored by implantable cardiac devices might significantly improve patient care, nocturnal respiratory data from these devices have not been fully validated.

The aim of this study was to evaluate the performance of the ApneaScan ${ }^{\mathrm{TM}}$ algorithm among patients who underwent PMK or ICD implantation according to standard indications.

\section{Methods}

\subsection{Study design and objectives}

The present study is a sub-analysis of the Multiparametric Heart Failure Evaluation in Internal Cardioverter Defibrillators (ICD) Patients (MULTITUDE-HF) trial [9]. Data from 31 consecutive patients scheduled for implantation of a PMK or an ICD endowed with the ApneaScan ${ }^{\text {TM }}$ diagnostic feature (Boston Scientific Inc., Natick, MA, USA) were prospectively collected in an institutional review boardapproved registry. All patients gave written informed consent for the diagnostic and invasive procedures. Devices and pacing leads were implanted by means of standard techniques. Eligibility for device implantation was based on international guidelines [10]. A home sleep monitoring (HSM) study (polygraphy) was prescribed to all patients 1 month following device implantation.

The device was interrogated on the morning after the sleep study and the average number of apnea/hypopnea events per hour, as detected by the ApneaScan ${ }^{\mathrm{TM}}$ algorithm throughout the same night of the HSM study, was documented.
This value, reported by the device as respiratory disturbance index (RDI), was subsequently compared with the apnea/hypopnea index (AHI) assessed by means of nocturnal polygraphy.

The objectives of the present study were to (1) assess the level of agreement between the device-calculated RDI and the AHI values measured by the HMS study; (2) calculate the RDI cutoff for maximization of sensitivity and specificity of device-detected SRBD; and (3) estimate the day-to-day variability of the RDI values measured by the device over a period of 7 days prior to the sleep study.

Moderate-to-severe chronic obstructive pulmonary disease, primary pulmonary hypertension, or a known diagnosis of severe SRBD were exclusion criteria of the study. Baseline clinical characteristics, drug therapy, ECG, and echocardiographic parameters were prospectively collected.

\subsection{Sleep study recording}

All patients enrolled in the study underwent a home sleep study recording by means of a multi-sensor portable sleep apnea monitor (Embletta, Broomfield, USA). Polygraphy results were scored by two experienced sleep experts (AR, MA), board certified by the Italian Association of Sleep Medicine, who were unaware of the results reported by the implantable cardiac device [11]. The parameters monitored during the sleep study included nasal and oral flow, air temperature, oxygen saturation via finger pulse oximetry, respiratory effort through plethysmography, snoring, body positions, and movements. Apnea was defined as a decrease in oronasal airflow $>90 \%$ lasting $\geq 10 \mathrm{~s}$; hypopnea was defined as a decrease in flow $>50 \%$ lasting $\geq 10$ s coupled with a $>3 \%$ drop in oxygen saturation [11]. The AHI was calculated as apnea/hypopnea episodes divided by the hours of sleep (sleep time). The sleep time did not include any movement or standing position times detected during the sleep study. SA was classified as normal for AHI $<5$, mild for AHI between 5 and 14.9, moderate for AHI between 15 and 29.9, and severe for $\mathrm{AHI} \geq 30[7,12]$.

\subsection{Device characteristics, SA detection, and analysis}

Patients were implanted with commercially available PMKs and ICDs with sleep respiratory disturbances monitoring capability (ApneaScan ${ }^{\mathrm{TM}}$ ).

The ApneaScan ${ }^{\mathrm{TM}}$ sensor continuously measures fluctuations in thoracic electrical impedance by sending a low-voltage signal from the lead and the can. Thoracic impedance changes occurring during inspiration and 
expiration are detected and used to create a respiratory waveform. The waveform is used to detect breaths and their relative tidal volumes. At night, the algorithm is able to identify apnea/hypopnea episodes by detecting reductions in the average tidal volume. An apnea/hypopnea event is counted if tidal volumes remain below $74 \%$ of the average value for $>10 \mathrm{~s}$ or no respiratory waveform is detected. The RDI is calculated as follows: overall number of apnea or hypopnea events/hours of sleep [7].

This datum is presented as a trend on pacemaker interrogation (Fig. 1).

The company suggests adopting an RDI value of 32 episodes $/ \mathrm{h}$ as a cutoff for severe SA.

\subsection{Statistical analysis}

Continuous data were expressed as mean \pm standard deviation (SD). Categorical data were expressed as percentages. Differences between mean data were compared by means of a $t$ test. Optimal cutoff values of parameters were determined by means of receiver-operating characteristic curve analysis. Agreement between the two indexes was computed as the bias (mean), with limits of agreement computed as bias $\pm 2 \mathrm{SD}$ when differences followed normal distribution. Normality was tested by means of the Kolmogorov-Smirnov one-sample test. A $p$ value $<0.05$ was considered significant for all tests. All statistical analyses were performed by means of STATISTICA software, version 7.1 (StatSoft, Inc.).

\section{Results}

\subsection{Study population}

A total of 31 consecutive patients with a standard indication for permanent pacing $(N=12)$ or $\operatorname{ICD}(N=19)$ underwent device implantation at our center and were enrolled in the study. The cohort was $64.5 \%$ male, with a mean age of $70 \pm 8$ years. Baseline characteristics of the population are reported in Table 1 .

\subsection{HSM and SA detection}

Stored RDI data were collected in the morning following the overnight HSM study.

A device-measured RDI value was not available in 2 patients during the study night and these patients were excluded from the analysis. In the remaining 29 patients, the mean HSM-AHI was $23 \pm 19$ episodes/h and 7 (24.1\%) of them had a diagnosis of severe SA (Fig. 2). The average RDI value documented by the sensor during the same night was $34 \pm 22$ episodes/h and was significantly higher in patients with severe SA on the sleep study recording ( $65 \pm 18$ versus $24 \pm 12$ episodes $/ \mathrm{h}, p<0.001$ ).

Receiver-operating characteristic curve analysis for the ability of the RDI to detect a severe form of SA revealed a sensitivity and specificity of an RDI value of 32 episodes $/ \mathrm{h}$ of $71 \%$ and $81 \%$, respectively. The cutoff value that best identified severe SA was 47 episodes/h. This enabled severe SA to be detected

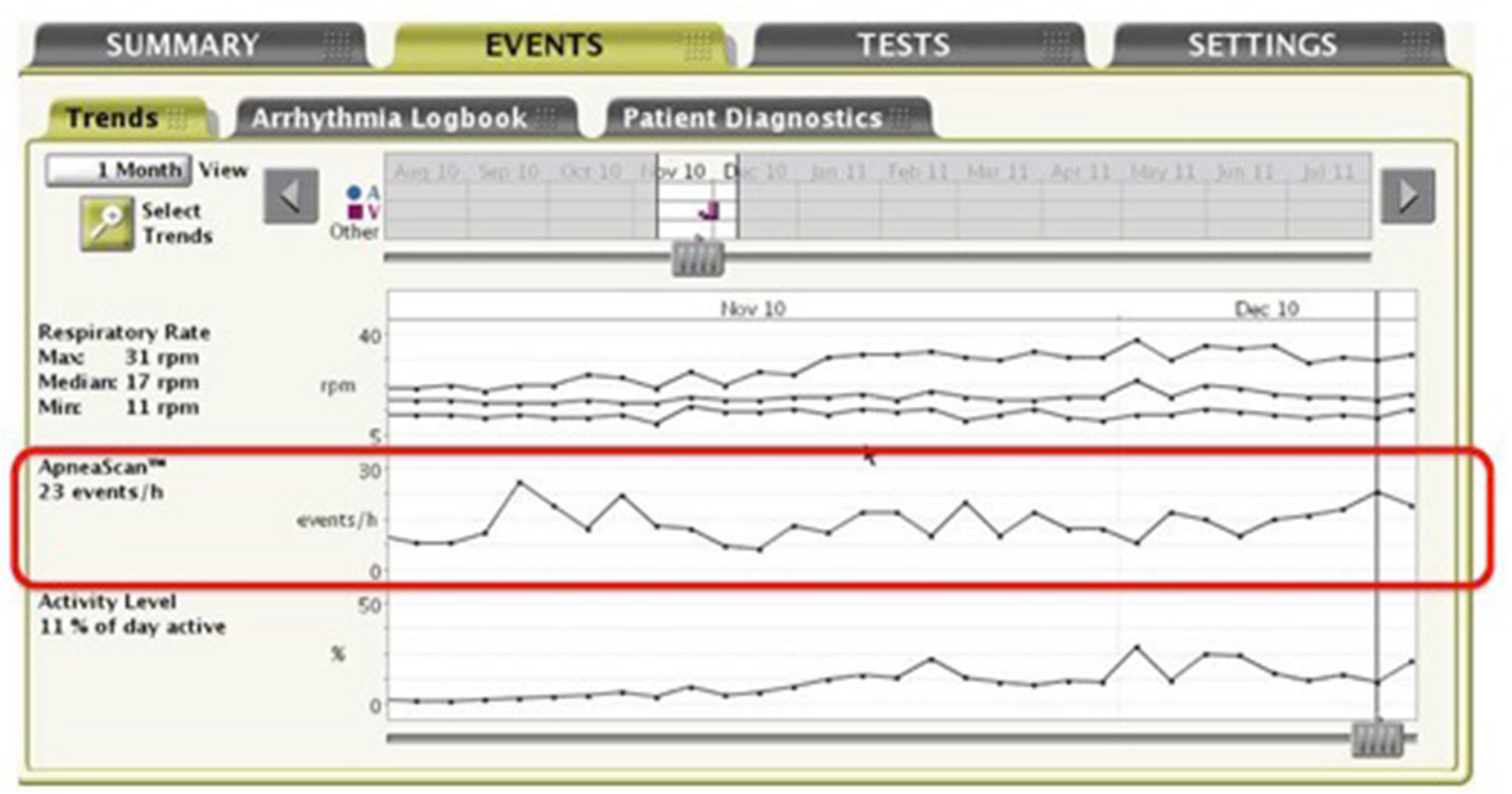

Fig. 1 The respiratory disturbance index is the average number of apnea/hypopnea events per hour throughout the night and is presented as a daily trend 
Table 1 Demographics and baseline clinical parameters

\begin{tabular}{ll}
\hline Parameter & $N=31$ \\
\hline Male gender $(n(\%))$ & $20(64.5)$ \\
Age (years) & $70 \pm 8$ \\
BMI & $29 \pm 4$ \\
Hypertension $(n(\%))$ & $7(22.6)$ \\
Diabetes $(n(\%))$ & $9(29.0)$ \\
Dyslipidemia $(n(\%))$ & $11(35.5)$ \\
COPD $(n(\%))$ & $7(22.6)$ \\
CKD $(n(\%))$ & $11(35.5)$ \\
LVEF $(\%)$ & $42 \pm 14$ \\
Coronary artery disease $(n(\%))$ & $12(38.7)$ \\
Dilated cardiomyopathy $(n(\%))$ & $16(51.6)$ \\
History of AF $(n(\%))$ & \\
Paroxysmal $(n(\%))$ & $9(29.0)$ \\
Persistent $(n(\%))$ & $1(3.2)$ \\
\hline
\end{tabular}

Values are reported as mean $\pm \mathrm{SD}$ or $n(\%) . A F$, atrial fibrillation; $B M I$, body mass index; $C K D$, chronic kidney disease; $C O P D$, chronic obstructive pulmonary disease; $L V E F$, left ventricular ejection fraction; TIA, transient ischemic attack

with $100 \%$ sensitivity and specificity. The Bland-Altman agreement analysis of AHI-RDI revealed a bias between measurements of -11 episodes/h, with limits of agreement -38 to 15 episodes/h (Fig. 3). The RDI value closely correlated with the AHI value $(r=0.80,95 \%$ CI 0.61 to $0.90 ; p<0.001)$.

Analysis of the RDI values measured by the device identified 14 patients with at least 2 RDI values collected over a period of 7 days prior to the sleep study. Individual maximum and minimum RDI values in these patients are reported in Fig. 4. The mean weekly RDI variability (maximum RDIminimum RDI) was $18 \pm 8$ episodes/h, and $5(36 \%)$ patients presented RDI values both below and above the cutoff value of 47 episodes/h during the week.

\section{Discussion}

In the present study, we investigated the accuracy of a novel transthoracic impedance-based $\mathrm{PMK} / \mathrm{ICD}$ sensor for automatic detection of SRBD. The device-calculated RDI value appeared to accurately identify severe SRBD. Good agreement was observed when comparing devicedetected RDI estimations and values of AHI assessed by means of nocturnal polygraphy. Moreover, the opportunity to continuously monitor the RDI allowed us to detect a wide day-to-day variability in the amount of respiratory disturbance. This critical issue is actually well known in sleep medicine; the AHI shows an important inter-individual variability, especially when applied in classification or as a criterion of therapeutic success during a single recording [13]. In this perspective, continuous monitoring of nocturnal apnea/hypopnea episodes becomes of utmost importance for diagnostic and therapeutic purposes.

Of note, SRBD are a common finding among patients with cardiovascular diseases [1].

Specifically, SRBD have been demonstrated to significantly increase the risk of developing hypertension [2] and arrhythmias [3, 4, 14], as well as worsen outcomes in patients with heart failure [5], being a predictor of premature cardiovascular death. The prevalence of both bradyarrhythmias and tachyarrhythmias is remarkably high in patients suffering from SRBD and increase with the number of apneic episodes and severity of hypoxemia [15]. Approximately 50\% of SRBD patients experience nocturnal arrhythmias, especially complex ventricular ectopy, sinus arrest, and atrio-ventricular conduction block [4]. Moreover, individuals with SRBD have up to four times the odds of atrial fibrillation (AF) compared with the general population [16] and obstructive SA in patients with AF represents an independent predictor of stroke [17]. Recently, devicediagnosed severe SA has been demonstrated to be independently associated with a higher risk of AF and new onset AF in PMK patients [18].

In patients with suspected SRBD, a definitive diagnosis should require a full-night laboratory nocturnal polysomnography (PSG) spending a night in a sleep laboratory [11], during which multiple polygraphic variables are continuously recorded (electroencephalogram, electromyogram, electro-oculogram, respiration, and snoring).
Fig. 2 Individual values of the respiratory disturbance index measured by the device during the sleep study night. Red dots: patients with severe SA during the sleep study (AHI $\geq 30$ episodes/ h); blue dots: patients with $\mathrm{AHI}<$ 30 episodes $/ \mathrm{h}$

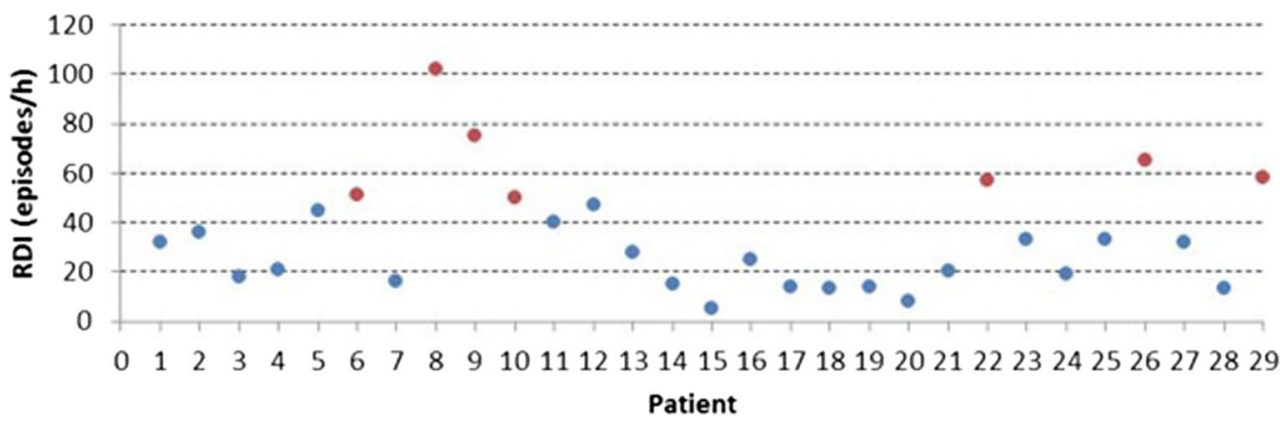




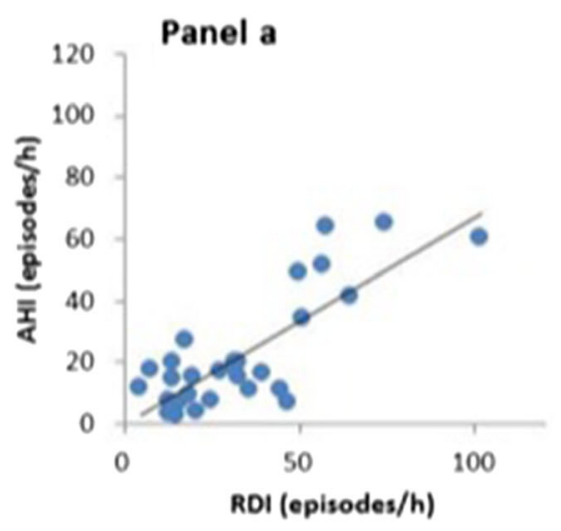

Fig. 3 Panel a Diagram showing the data pairs in all 29 patients. Panel b Bland-Altman plots. AHI-RDI is the difference between indexes; average

Although PSG is considered the diagnostic gold standard, access to this procedure is limited, as it requires special institutions and trained technicians and is relatively expensive. In addition, as previously reported, a single recording may be biased by intra-individual variability [13].

Although laboratory full PSG is considered the investigation of choice to diagnose SRBD, it is expensive and problematic for patients who have to travel to a center and spend a night away from home. HSM represents a validated, close to the real life, and less expensive tool to diagnose SA [19]. In addition, there is growing evidence that home devices may be useful in several clinical settings particularly when SRDB is suspected [20]. However, HSM, though generally effective, may underestimate the SA severity because of the differences in methods to detect obstructive events and amount of sleep. The numerator of the AHI may be lower for a portable test than an in-laboratory test, as subtle sleep-disordered breathing is not as easily identified, as it would on an inlaboratory test because of the inability to detect arousalrelated events (so-called respiratory effort-related arousals). Additionally, the denominator (time) is larger with portable tests because recording time is assessed rather than the real sleep time (lacking EEG signal for sleep scoring in HSM) [19].
Even if HSM simplifies the diagnostic process, a remarkably high number of patients with severe SA still remains undiagnosed and/or undertreated [21]. As an example, 24\% of patients in our cohort were newly diagnosed with severe SA. The rate of patients with undiagnosed, clinically significant SRBD has been reported to be as high as $85 \%$ [22].

Some recent PMKs and ICDs feature thoracic impedance monitoring systems, which allow the device to detect fluctuations in tidal volume occurring in SRBD [7]. The aim of our study was to validate the diagnostic accuracy of the ApneaScanTM algorithm to detect and monitor sleep respiratory disturbances.

The ability of the algorithm to identify patients with severe sleep-disordered breathing seemed high, thus necessitating further investigation of the severity and form of SA severity, and possibly additional therapy. Although there was good agreement between HSM-AHI and ApneaScan ${ }^{\mathrm{TM}}$-RDI measurements recorded during the sleep study night, this device feature should be considered for screening purposes only and not as an alternative to home or laboratory sleep monitoring studies. We found higher values of ApneaScan ${ }^{\mathrm{TM}}$-RDI when compared with nocturnal monitoring values. Although ApneaScan ${ }^{\mathrm{TM}}$ is considered a simple screening tool requiring diagnostic confirmation, this issue may be explained also by the
Fig. 4 Individual maximum and minimum of RDI values measured by the device during the week preceding the study night. The red stars indicate patients with RDI values both below and above the cutoff value of 47 episodes/h during the week

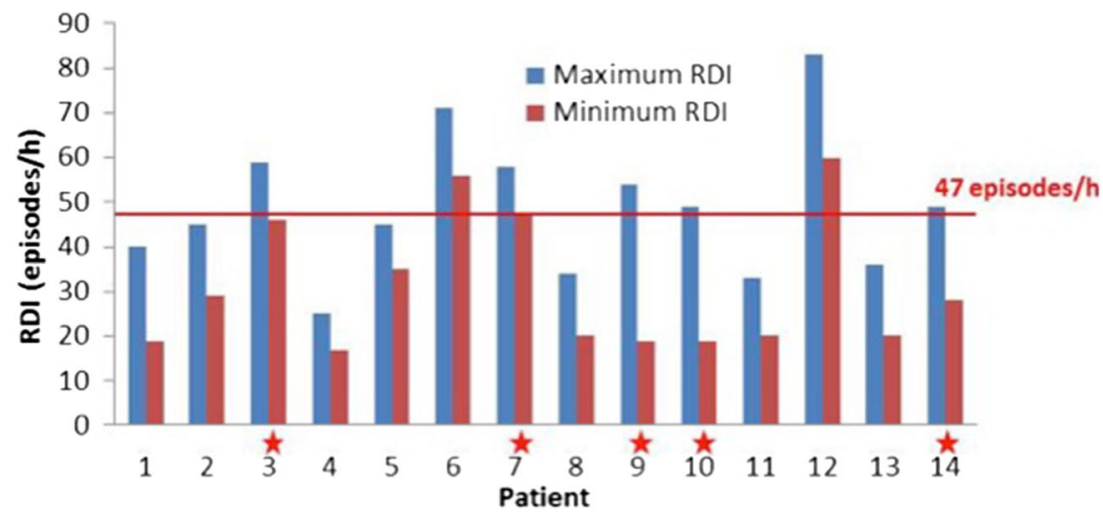


AHI underestimation obtained from HSM versus full PSG as previously reported. Nevertheless, ApneaScan ${ }^{\mathrm{TM}}$ may be considered an efficient tool for pre-screening patients who require additional investigation, within the framework of a complete management program, even if full PSG may confirm or increase its potential use.

The availability of a tool for the automatic and continuous measurement of SA could enable their long-term behavior to be analyzed, thereby allowing the possible effect of therapeutic interventions to be monitored. In our analysis, we observed wide day-to-day variability in the severity of SA. Specifically, in $36 \%$ of patients in whom multiple measurements were taken over a period of 7 days prior to the sleep study, we recorded RDI values both below and above the identified cutoff for severe SA. Similar observations of a wide intra-individual variability in AHI/RDI values were reported in a series of patients undergoing multiple polysomnographic recordings [23] at different time points (mean interval 3.3 weeks), as well as in a cohort of 58 patients [24] with PMKs equipped with a respiratory sensor from a different brand (mean intraindividual day-to-day RDI variability $19 \% \pm 21 \%$ ). In our opinion, this preliminary result seems to question the clinical validity of a single assessment by means of PSG, in that the variability of the value overtime could result in inaccurate diagnoses and therapeutic decisions.

\subsection{Limitations}

The study has some potential limitations, which need to be addressed. First, the main limitation of the present study is the low number of patients included. Second, the algorithm for SA detection does not distinguish between obstructive and central SA. Therefore, despite its accuracy in detecting SA, its adoption in clinical practice will probably remain limited to the preliminary screening of possible respiratory disturbances, which means that dedicated monitors will not fully replace sleep studies. Third, a safety feature of the algorithm is to interrupt for 1 hour any respiratory sensor recordings if three or more rapid ventricular intervals are registered on the ventricular channel. In patients with frequent runs of non-sustained ventricular ectopies, missing data can result from arrhythmia-related sensor inhibition, therefore hindering night-to-night RDI variability analysis.

\subsection{Conclusion}

The ability of the ApneaScan ${ }^{\mathrm{TM}}$ algorithm to identify patients with SRBD seemed high, thus requiring further investigation of the severity and form of apnea, and possibly necessitating additional therapy.

\section{Compliance with ethical standards}

Conflict of interest Lovecchio $\mathrm{M}$ and Valsecchi $\mathrm{S}$ are employees of Boston Scientific. Di Biase L is a consultant for Biosense Webster, Boston Scientific, Stereotaxis, and St. Jude Medical and has received speaker honoraria from Medtronic, Atricure, EPiEP, and Biotronik. Natale A has received speaker honoraria from Boston Scientific, Biosense Webster, St. Jude Medical, Biotronik, and Medtronic and is a consultant for Biosense Webster, St. Jude Medical, and Janssen. The remaining authors have no disclosures.

Ethical approval All procedures performed in studies involving human participants were in accordance with the ethical standards of the institutional and/or research committee and with the 1964 Helsinki Declaration and its later amendments or comparable ethical standards. The study is a sub-analysis of the Institutional Review Board-approved registry Multiparametric Heart Failure Evaluation in Internal Cardioverter Defibrillators (ICD) Patients (MULTITUDE-HF) (ClinicalTrials.gov Identifier: NCT01501331).

\section{References}

1. Somers VK, White DP, Amin R, Abraham WT, Costa F, Culebras A, et al. American Heart Association Council for High Blood Pressure Research Professional Education Committee, Council on Clinical Cardiology; American Heart Association Stroke Council; American Heart Association Council on Cardiovascular Nursing; American College of Cardiology Foundation. Sleep apnoea and cardiovascular disease: an American Heart Association/American College Of Cardiology Foundation Scientific Statement from the American Heart Association Council for High Blood Pressure Research Professional Education Committee, Council on Clinical Cardiology, Stroke Council, and Council On Cardiovascular Nursing. In collaboration with the National Heart, Lung, and Blood Institute National Center on Sleep Disorders Research (National Institutes of Health). Circulation. 2008;118:1080-11.

2. Peppard PE, Young T, Palta M, Skatrud J. Prospective study of the association between sleep-disordered breathing and hypertension. N Engl J Med. 2000;342:1378-84.

3. Tang RB, Dong JZ, Liu XP, Kang JP, Ding SF, Wang L, et al. Obstructive sleep apnoea and the recurrence of atrial fibrillation. Circulation. 2003;107:2589-94.

4. Fichter J, Bauer D, Arampatzis S, Fries R, Heisel A, Sybrecht GW. Sleep-related breathing disorders are associated with ventricular arrhythmias in patients with an implantable cardioverter-defibrillator. Chest. 2002;122:558-61.

5. Lanfranchi PA, Somers VK, Braghiroli A, Corra U, Eleuteri E, Giannuzzi P. Central sleep apnoea in left ventricular dysfunction: prevalence and implications for arrhythmic risk. Circulation. 2003;107:727-32.

6. Defaye P, Pépin JL, Poezevara Y, Mabo P, Murgatroyd F, Lévy P, et al. Automatic recognition of abnormal respiratory events during sleep by a pacemaker transthoracic impedance sensor. J Cardiovasc Electrophysiol. 2004;15:1034-40.

7. Shalaby A, Atwood C, Hansen C, Konermann M, Jamnadas P, Lee $\mathrm{K}$, et al. Feasibility of automated detection of advanced sleep disordered breathing utilizing an implantable pacemaker ventilation sensor. Pacing Clin Electrophysiol. 2006;29:1036-43.

8. Defaye P, de la Cruz I, Martí-Almor J, Villuendas R, Bru P, Sénéchal J, et al. A pacemaker transthoracic impedance sensor with an advanced algorithm to identify severe sleep apnoea: the DREAM European study. Heart Rhythm. 2014;11:842-8. 
9. Forleo GB, Santini L, Campoli M, Malavasi M, Scaccia A, Menichelli M, et al. Long-term monitoring of respiratory rate in patients with heart failure: the Multiparametric Heart Failure Evaluation in Implantable Cardioverter-Defibrillator Patients (MULTITUDE-HF) study. J Interv Card Electrophysiol. 2015;43(2):135-44.

10. Priori SG, Blomström-Lundqvist C, Mazzanti A, Blom N, Borggrefe M, Camm J, et al. 2015 ESC Guidelines for the management of patients with ventricular arrhythmias and the prevention of sudden cardiac death: the Task Force for the Management of Patients with Ventricular Arrhythmias and the Prevention of Sudden Cardiac Death of the European Society of Cardiology (ESC). Endorsed by: Association for European Paediatric and Congenital Cardiology (AEPC). Europace. 2015;17:1601-87.

11. Berry RB, Budhiraja R, Gottlieb DJ, Gozal D, Iber C, Kapur VK, et al. American Academy of Sleep Medicine. Rules for scoring respiratory events in sleep: update of the 2007 AASM Manual for the Scoring of Sleep and Associated Events. Deliberations of the Sleep Apnea Definitions Task Force of the American Academy of Sleep Medicine. J Clin Sleep Med. 2012;8:597-619.

12. Sleep-related breathing disorders in adults: recommendations for syndrome definition and measurement techniques in clinical research. The Report of an American Academy of Sleep Medicine Task Force. Sleep. 1999;22:667-89.

13. Bittencourt LR, Suchecki D, Tufik S, Peres C, Togeiro SM, Bagnato MC, et al. The variability of the apnoea-hypopnoea index. J Sleep Res. 2001;10:245-51.

14. Zeidan-Shwiri T, Aronson D, Atalla K, Blich M, Suleiman M, Marai I, et al. Circadian pattern of life-threatening ventricular arrhythmia in patients with sleep-disordered breathing and implantable cardioverter-defibrillators. Heart Rhythm. 2011;8:657-62 .

15. Koehler U, Schäfer H. Is obstructive sleep apnoea a risk factor for myocardial infarction and cardiac arrhythmias in patients with coronary heart disease? Sleep. 1996;19:283-6.
16. Gami AS, Pressman G, Caples SM, Kanagala R, Gard JJ, Davison $\mathrm{DE}$, et al. Association of atrial fibrillation and obstructive sleep apnoea. Circulation. 2004;110:364-7.

17. Yaranov DM, Smyrlis A, Usatii N, Butler A, Petrini JR, Mendez J, et al. Effect of obstructive sleep apnoea on frequency of stroke in patients with atrial fibrillation. Am J Cardiol. 2015;115:461-5.

18. Mazza A, Bendini MG, De Cristofaro R, Lovecchio M, Valsecchi S, Boriani G. Pacemaker-detected severe sleep apnea predicts newonset atrial fibrillation. Europace. 2017;19(12):1937-43.

19. Collop NA, Anderson WM, Boehlecke B, Claman D, Goldberg R, Gottlieb DJ, et al. Portable Monitoring Task Force of the American Academy of Sleep Medicine. Clinical guidelines for the use of unattended portable monitors in the diagnosis of obstructive sleep apnoea in adult patients. Portable Monitoring Task Force of the American Academy of Sleep Medicine. J Clin Sleep Med. 2007;3:737-47.

20. Romigi A, Vitrani G, D'Aniello A, Di Gennaro G. Can nocturnal groaning be suspected by cardiorespiratory polygraphy? Sleep Med. 2014;15:1002-4.

21. Flemons WW, Douglas NJ, Kuna ST, Rodenstein DO, Wheatley J. Access to diagnosis and treatment of patients with suspected sleep apnoea. Am J Respir Crit Care Med. 2004;169:668-72.

22. Kapur V, Strohl KP, Redline S, Iber C, O’Connor G, Nieto J. Underdiagnosis of sleep apnoea syndrome in U.S. communities. Sleep Breath. 2002;6:49-54.

23. Aarab G, Lobbezoo F, Hamburger HL, Naeije M. Variability in the apnea-hypopnea index and its consequences for diagnosis and therapy evaluation. Respiration. 2009;77:32-7.

24. Moubarak G, Bouzeman A, de Geyer d'Orth T, Bouleti C, Beuzelin C, Cazeau S. Variability in obstructive sleep apnea: analysis of pacemaker-detected respiratory disturbances. Heart Rhythm. 2017;14:359-64.

Publisher's note Springer Nature remains neutral with regard to jurisdictional claims in published maps and institutional affiliations. 